EESTI NSV TEADUSTE AKADEEMIA TOIMETISED. 31. KOIDE

FOOSIKA * MATEMAATIKA. 1982, NR. 3

ИЗВЕСТИЯ АКАДЕМИИ НАУК ЭСТОНСКОИ ССР. ТОМ 31

ФИЗИКА * МАТЕМАТИКА, 1982, № 3

удк 539.3

A. PABACOO

\title{
РАСПРОСТРАНЕНИЕ ВОЛН В СРЕДЕ С НЕОДНОРОДНОЙ НАЧАЛЬНОЙ СТАТИЧЕСКОЙ ДЕФОРМАЦИЕЙ
}

\author{
(Представил Н. Алумяэ)
}

Влияние однородной начальной статической деформации на скорости распространения волн в твердой среде исследовалось во многих работах (см., напр. $\left.\left[{ }^{1-5}\right]\right)$. Выведены зависимости изменения скоростей волн от деформации - акустоупругие коэффициенты, величина которых зависит от типа волн и от направления их распространения. В случае неоднородных начальных деформаций вывод этих зависимостей значительно осложняется, поскольку определение скорости волн связано с решением дифференциальных уравнений в частных производных с переменными коэффициентами. Этим объясняется недостаточная изученность проблемы акустодиагностики таких сред. Ниже рассматривается распространение продольной волны в твердой среде, подвергнутой неоднородной плоской деформации, и обсуждаются вопросы акустодиагностики этой среды. Выводится решение типа интеграла свертки, которое позволяет описать профиль продольной волны, возбужденной краевым воздействием произвольной формы в твердой среде, находящейся в состоянии плоской деформации. Более подробно исследуется случай распространения синусоидальной волны в среде, подвергнутой чистому изгибу со сжатием или растяжением в направлении, перпендикулярном оси распространения волны. Задача акустодиагностики начального состояния среды решается путем регистрации амплитуды волны и времени ее прибытия в точку наблюдения.

1. Исследуемая среда описывается формулами нелинейной теории упруго деформируемых твердых сред $\left.\Gamma^{6}\right]$. Используется лагранжева система декартовых координат $X_{1}, X_{2}, X_{3}$.

Предполагая, что производные от перемещения материальных точек среды

$$
\left|U_{i, j}\right| \ll 1,
$$

разложим функцию внутренней энергии $W$ в ряд относительно естественного состояния [ $\left.{ }^{6}\right]$

$$
\mathrm{Q}_{0} W=\frac{1}{2} \lambda K_{1}^{2}+\mu K_{2}+v_{1} K_{1}^{3}+v_{2} K_{1} K_{2}+v_{3} K_{3}+O\left(E_{i j}^{4}\right),
$$

где $\varrho_{n}$ - плотность среды в естественном недеформированном состоянии, $E_{i j}$ - тензор деформации Лагранжа, $K_{j}$ - инварианты тензора деформации $E_{i j}, \lambda, \mu$ - модули упругости второго порядка, $v_{i}$ - модули упругости третьего порядка, $i, j=1,2,3$. Далее $t-$ время и используется обозначение производных

$$
U_{i, j}=\frac{\partial U_{i}}{\partial X_{j}}, \quad U_{i, t}=\frac{\partial U_{i}}{\partial_{t}} .
$$

Уравнение движения среды имеет вид 


$$
\sigma_{i j, j}=\mathrm{Q}_{0} U_{i, t t} .
$$

Используя выражение для тензора псевдонапряжения Лагранжа $\sigma_{i j}$ $\left[{ }^{6}\right]$, представим уравнение движения среды в перемещениях

$$
\begin{gathered}
\left(\lambda+\mu+6 v_{1} U_{m, m}+v_{2} U_{n, n}\right) U_{m, m i}+\left[\mu+\left(\lambda+v_{2}\right) U_{n, n}\right] U_{i, m m}+ \\
+\left(\lambda+\mu+v_{2}+3 v_{3} / 4\right)\left(U_{n, m} U_{n, m i}+U_{i, m} U_{n, m n}\right)+ \\
+\left(v_{2}+3 v_{3} / 4\right)\left(U_{m, n} U_{n, m i}+U_{n, i} U_{m, m n}\right)+\left(\mu+3 v_{3} / 4\right)\left[\left(U_{n, m}+U_{m, n}\right) U_{i, m n}+\right. \\
\left.+\left(U_{m, i}+U_{i, m}\right) U_{m, n n}\right]+O\left(U_{m, n}^{3-}\right)=\varrho_{0} U_{i, t t} .
\end{gathered}
$$

Далее рассмотрим плоское деформированное состояние среды, когда

$$
X_{1}=X_{1}\left(x_{1}, x_{2}, t\right), \quad X_{2}=X_{2}\left(x_{1}, x_{2}, t\right), \quad X_{3}=x_{3} .
$$

Здесь $x_{i}$ - эйлеровы переменные в эйлеровой декартовой системе координат, которая совпадает в момент времени $t=0$ с описанной выше лагранжевой.

Аналогично $\left[{ }^{1,2,5}\right]$ будем различать три состояния среды: естественное недеформированное, начальное деформированное и состояние среды в данный момент времени, допуская, что последнее получается из начального под воздействием дополнительного возмущения. Величины, относящиеся ,к начальному состоянию, будем отмечать верхним индексом «нуль», величины, характеризующие состояние среды в данный момент времени, - звездочкой сверху и величины, относящиеся к возмушению. оставим без индексов. Тогда имеет место соотношение $U_{i}^{*}=$ $=U_{i}{ }^{0}+U_{i}$.

Учитывая, что в плоском деформированном состоянии $U_{3}=0$ и что начальное деформированное состояние среды является статическим. уравнения движения можем упростить. Для их аналитического решения сделаем следующие допущения:

$$
\begin{gathered}
U_{1}=U_{1}\left(X_{1}, X_{2}, t\right), \quad\left|U_{1,2}\right| \ll\left|U_{1,1}\right|, \quad\left|U_{1,12}\right| \ll\left|U_{1,11}\right|, \\
\left|U_{2}\right| \ll\left|U_{1}\right|, \quad\left|U_{2, i}\right| \ll\left|U_{1, i}\right| .
\end{gathered}
$$

Это означает. что ниже исследуется прощесс распространения продольной волны вдоль оси $X_{1}$.

Учитывая только члены с порядком величин $U_{1,1}$ и $U_{1,11}$, придадим уравнениям движения (1.3) вид.

$$
\begin{gathered}
\left(1+k_{1} U_{1,1}^{0-}+k_{2} U_{2,2}^{0}\right) U_{1,11}+k_{1} U_{1,1} U_{1,11}+ \\
+\left[k_{1} U_{1,11}^{0}+k_{3} U_{1,22}^{0}+\left(k_{2}+k_{4}\right) U_{2,21}^{0}\right] U_{1,1}=c^{-2} U_{1, t t} \\
\left(k_{3} U_{2,1}^{0}+k_{4} U_{1,2}^{0}\right) U_{1,11}+\left(k_{3} U_{2,1}+k_{4} U_{1,2}\right) U_{1,11}+ \\
+\left[k_{2} U_{2,22}^{0-}+k_{3} U_{2,11}^{0}+\left(k_{4}+k_{5}\right) U_{1,12}^{0}\right] U_{1,1}=c^{-2} U_{2, t t},
\end{gathered}
$$

где

$$
\begin{aligned}
& k_{1}=3+6 k\left(v_{1}+v_{2}+v_{3}\right), \quad k_{2}=k\left(\lambda+2 v_{2}\right), \\
& k_{3}=1+k\left(v_{2}+3 v_{3} / 2\right), \quad k_{4}=k\left(\mu+v_{2}+3 v_{3} / 2\right), \\
& k_{5}=k\left(\lambda+6 v_{1}+2 v_{2}\right), \quad c^{-2}=\varrho_{0} k, \quad k=(\lambda+2 \mu)^{-1} .
\end{aligned}
$$

Уравнение (1.6) описывает распространение продольной волны в направлении оси $X_{1}$ в среде с начальной деформацией, а уравнение (1.7) позволяет оценить расширение зоны возмущения в направлении оси $X_{2}$. 
2. Уравнения (1.6) и (1.7) выведены с учетом неравенств

$$
\left|U_{i, i}^{0}\right| \ll 1, \quad\left|U_{i, i}\right| \ll 1 .
$$

В случае $U_{1,1}<U_{1,1}^{0}$ уравнение (1.6) можно линеаризировать, полагая, что из двух членов $k_{1} U_{1,1}^{0} U_{1,11}$ и $K_{1} U_{1,1} U_{1,11}$ последний является величиной высшего порядка малости по сравнению с единицей. Волновой процесс в среде описывается теперь уравнением

$$
f_{1}\left(X_{1}, X_{2}\right) U_{1,11}+f_{2}\left(X_{1}, X_{2}\right) U_{1,1}-c^{-2} U_{1, t t}=0,
$$

где

$$
\begin{gathered}
f_{1}\left(X_{1}, X_{2}\right)=1+k_{1} U_{1,1}^{0}+k_{2} U_{2,2}^{0}, \\
f_{2}\left(X_{1}, X_{2}\right)=k_{1} U_{1,11}^{0}+\left(k_{2}+k_{4}\right) U_{2,12}^{0}+k_{3} U_{1,22}^{0} .
\end{gathered}
$$

Уравнение (2.1) решается при нулевых начальных условиях

$$
U_{1}\left(X_{1}, 0\right)=0, \quad U_{1, t}\left(X_{1}, 0\right)=0
$$

и при краевых условиях

$$
\begin{gathered}
U_{1, t}(0, t)=-\varepsilon c \varphi(t) H(t), \\
U_{1}(\infty, t)=0 .
\end{gathered}
$$

Здесь $H(t)$ - функция Хевисайда, $\varphi(t)$ - произвольная функция и $\varepsilon-$ постоянная. $\varphi(t)$ и $\varepsilon$ удовлетворяют условиям

$$
\max |\varphi(t)|=1, \quad 0<\varepsilon \ll 1 .
$$

Поскольку исследуется распространение волн в среде, начальное деформированное состояние которой статическое, то коэффициенты $f_{1}$ и $f_{2}$ уравнения $(2.1)$ не зависят от времени. Применяя преобразование Лапласа по времени и учитывая (2.2), получим уравнение

$$
f_{1} U_{1,11}^{L}+f_{2} U_{1,1}^{L}-p^{2} c^{-2} U_{1}^{L}=0 .
$$

Последнее решается при краевых условиях (2.3) и (2.4) ВКБ-методом. Подстановкой

$$
U^{L}=C \exp \left\{\int\left[p u-f_{2}\left(2 f_{1}\right)^{-1}\right] d X_{1}\right\}
$$

уравнение (2.5) сводится к уравнению Риккати

$$
p u_{, 1}+p^{2} u^{2}-p^{2} f-g=0,
$$

где

$$
\begin{array}{ll}
f=c^{-2} f_{1}^{-1}, & g=f_{3}^{2}+f_{3,1}, \\
f_{3}=\frac{1}{2} f_{1}^{-1} f_{2}, & f_{i, j}=\frac{\partial f_{i}}{\partial X_{j}} .
\end{array}
$$

Решение уравнения Риккати ищется в виде ряда

$$
u=\sum_{k=0}^{\infty} u_{k} p^{-k}
$$

подставляя который в уравнение (2.6) и сравнивая коэффициенты при одинаковых степенях $p$, получим

$$
u_{0}= \pm \sqrt{f}
$$




$$
\begin{aligned}
& u_{1}=-f_{, 1}(4 f)^{-1}, \\
& u_{2}= \pm(2 \sqrt{f})^{-1}\left[g+f_{, 11}(4 f)^{-1}-5 f_{, 1}^{2}\left(16 f^{2}\right)^{-1}\right] .
\end{aligned}
$$

Сохраняя в ряде (2.7) первые три члена, получим решение преобразованного уравнения (2.5) в виде

$$
U_{1}^{L}=-\varepsilon c \varphi^{L} p^{-1} \exp \Phi_{1} \exp \left[-p \Phi_{2}-p^{-1} \Phi_{3}\right]
$$

и решение соответствующего ему исходного уравнения (2.1) в виде

$$
U_{1}=-\varepsilon c H(\zeta) \exp \Phi_{1} \int_{0}^{\zeta} \varphi(\zeta-\xi) J_{0}\left(2 \sqrt{\xi \Phi_{3}}\right) d \xi
$$

где

$$
\begin{aligned}
& \zeta=t-\Phi_{2} \\
& \Phi_{1}=-\frac{1}{4} \int_{0}^{X_{1}} f_{1}^{-1}\left(2 f_{2}-f_{1,1}\right) d X \\
& \Phi_{2}=\int_{0}^{X_{1}} \sqrt{f} d X \\
& \Phi_{3}=\frac{1}{2} \int_{0}^{X_{1}} f^{-1 / 2}\left(g+\frac{1}{4} f_{, 11} f^{-1}-\frac{5}{16} f_{, 1}^{2} f^{2}\right) d X
\end{aligned}
$$

Выражение (2.8) позволяет определить эволюцию краевого возмущения произвольной формы в среде с начальной статической деформацией. При выводе (2.8) использован ряд (2.7), который сходится при больших значениях параметра $p$, что означает справедливость (2.8) при

$$
\zeta \ll 1,
$$

т. е. в некоторой прифронтовой зоне.

Поскольку $\zeta=t-\Phi_{2}$, для определения состояния начальной деформации среды на основе полученного результата (2.8) в нашем распоряжении есть три функции $\Phi_{1}, \Phi_{2}, \Phi_{3}$, из которых $\Phi_{1}$ характеризует уменьшение амплитуды краевого возмущения, $\Phi_{2}-$ время прибытия фронта продольной волны в точку наблюдения, а $\Phi_{3}-$ искажение формы волны. Следовательно, из одного эксперимента можно определить значения трех параметров состояния начальной деформации среды. При этом скорость распространения волны определяется зависимостью

$$
c^{*}=X_{1} \Phi_{2}^{-1} .
$$

Рассмотрим конкретный пример, когда форма возмущения задана в виде

$$
\varphi(t)=\sin \omega t
$$

где $\omega-$ угловая частота возмущения.

Подставляя выражение (2.11) в уравнение (2.8) и используя стандартное разложение

$$
J_{0}\left(2 \sqrt{\Phi_{3} \xi}\right)=\sum_{k=0}^{\infty}(-1)^{k}(k !)^{-2}\left(\Phi_{3} \xi\right)^{k}
$$

вычислим интеграл свертки в виде ряда. Окончательный результат примет вид 


$$
\begin{aligned}
U_{1, t}=-\varepsilon c H(\zeta) & \exp \Phi_{1}\left\{\sin \omega \zeta+\sum_{k=1}^{\infty}(-1)^{k} \Phi_{3}^{k} \omega^{-k}(k !)^{-1}[\sin (\omega \zeta-k \pi / 2)+\right. \\
+ & \left.\left.\sum_{m=0}^{k}(\zeta \omega)^{k-m-1}((k-m-1) !)^{-1} \cos (m \pi / 2)\right]\right\} \\
& U_{1}=\varepsilon c \omega^{-1} H(\zeta) \exp \Phi_{1}\{\cos \omega \zeta-1+ \\
+ & \sum_{k=1}^{\infty}(-1)^{k} \Phi_{3}^{k}(k !)^{-1} \omega^{-k}[\cos (\omega \zeta-k \pi / 2)- \\
& \left.\left.-\sum_{m=0}^{k}(\zeta \omega)^{k-m}((k-m) !)^{-1} \cos (m \pi / 2)\right]\right\}
\end{aligned}
$$

Легко доказать, что единственное ограничение на решения (2.13) и (2.14) накладывает неравенство (2.9), поскольку в практических приложениях условие

$$
\left|\Phi_{3 \omega^{-1}}\right| \ll 1
$$

выполняется.

3. Рассмотрим конкретный случай состояния неоднородной начальной деформации среды, предстагляющий практический интерес. По сведениям автора, эта задача стандартными методами акустоупругости еще не решалась $[1,5]$.

Пусть начальное деформированное состояние среды описывается выражениями

$$
\begin{aligned}
& U_{1}^{0}=-\alpha X_{1}\left(a+b X_{1} / 2\right)-\beta b X_{2}^{2} / 2, \\
& U_{2}^{0}=\beta X_{2}\left(a+b X_{1}\right),
\end{aligned}
$$

где $a$ и $b-$ размерные коэффициенты и

$$
\alpha=\lambda[4 \mu(\lambda+\mu)]^{-1}, \quad \beta=(\lambda+2 \mu)[4 \mu(\lambda+\mu)]^{-1} .
$$

Выражения (3.1) удозлетворяют статическим уравнениям равновесия (уравнению (1.3) при $U_{i, t t}=0$ ). В этом случае компоненты тензора псевдонапряжения Лагранжа с точностью до линейных членов имеют вид

$$
\sigma_{22}^{0}=a+b X_{1}, \quad \sigma_{11}^{0}=\sigma_{12}^{0}=\sigma_{21}^{0}=0,
$$

что соответствует случаю, когда среда подвергнута изгибу с растяжением или сжатием по оси $X_{2}$ (см. рисунок).

Функции $\Phi_{1}, \Phi_{2}$ и $\Phi_{3}$ определяются с учетом выражения (3.1) следующим образом:

$$
\begin{aligned}
& \Phi_{\mathrm{t}}=b X_{1}\left(l_{1}+2 l_{2}\right)\left[1+l_{1} \theta\left(a+b X_{1} / 2\right)\right] / 4, \\
& \Phi_{2}=c^{-1} X_{1}\left[1+l_{1}\left(a+b X_{1} / 2\right) / 2\right] \\
& \Phi_{3}=-c b^{2} \eta X_{1}\left[1-\vartheta\left(a+b X_{1} / 2\right)\right]
\end{aligned}
$$

где

$$
\begin{aligned}
& \eta=\left(9 l_{1}^{2}-4 l_{2}^{2}\right) / 32, \quad \theta=\left(3 l_{1}+2 l_{2}\right)\left(l_{1}+2 l_{2}\right)^{-1} \\
& \vartheta=l_{1}\left(45 l_{1}^{2}+32 l_{1} l_{2}+12 l_{2}^{2}\right)\left(9 l_{1}^{2}-4 l_{2}^{2}\right)^{-1} / 2 \\
& l_{1}=[2 \mu(\lambda+\mu)]^{-1}\left[\lambda+v_{2}+3 \lambda\left(v_{1}+v_{2}+v_{3}\right)(\lambda+2 \mu)^{-1}\right] \\
& l_{2}=(4 \mu)^{-1}
\end{aligned}
$$




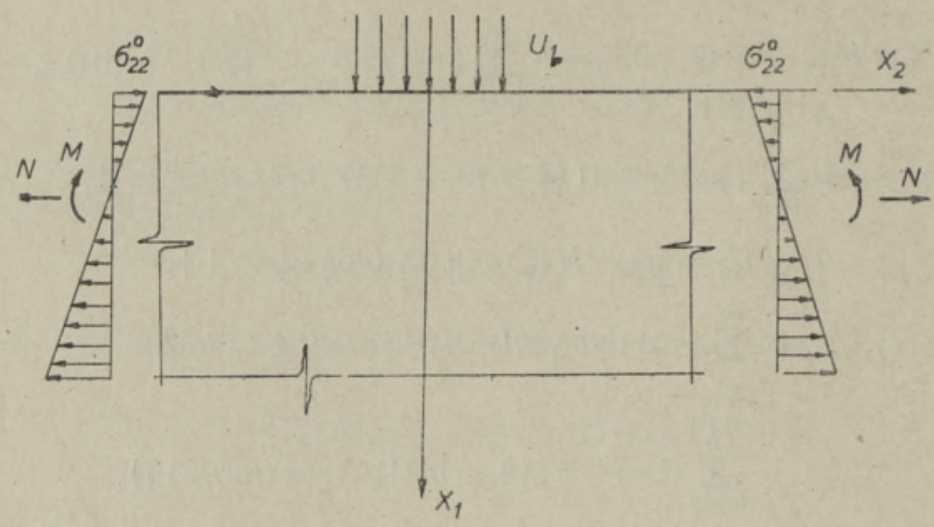

Подстановкой выражений (3.3) в (2.13) или (2.14) найдем зависимости, позволяющие определить искажение исходной формы волны в любой точке среды.

В случае, когда

$$
\left|\Phi_{3} \omega^{-1}\right| \ll 1
$$

выражения (2.13) и (2.14) можно использовать в виде

$$
\begin{gathered}
U_{1, t}=-\varepsilon c H(\zeta) \exp \Phi_{1} \sin \omega \zeta, \\
U_{1}=\varepsilon c \omega^{-1} H(\zeta) \exp \Phi_{1}(\cos \omega \zeta-1) .
\end{gathered}
$$

Теперь по данным эксперимента подлежат определению две функции $\Phi_{1}$ и $\Phi_{2}$; состояние начальной деформации среды определялось тоже двумя независимыми параметрами $a$ и $b$ (см. (3.1)). Если через $X_{1}{ }^{+}$обозначить координату $X_{1}$ точки наблюдения за искажением формы продольной волны и из эксперимента найти величины $\Phi_{1}$ и $\Phi_{2}$, то решение поставленной задачи дадут формулы

$$
\begin{aligned}
& a=2\left(l_{1} X_{1}^{+}\right)^{-1}\left(c \Phi_{2}-X_{1}^{+}\right)-b X_{1}^{+} / 2, \\
& b=4 \Phi_{1}\left[\left(l_{1}+2 l_{2}\right)\left(2 c \Phi_{2}-X_{1}^{+}\right)\right]^{-1},
\end{aligned}
$$

т. е. измерения определят искомые параметры $a$ и $b$.

Легко определить также скорость распространения волны в рассматриваемой среде

$$
c^{*}=c\left[1+l_{1}\left(a+b X_{1} / 2\right) / 2\right]^{-1},
$$

где $c$ - скорость распространения волны в недеформированной среде.

В хорошо изученном случае однородной начальной статической деформации, когда $b=0$, т. е. когда среда испытывает в направлении $X_{2}$ только растяжение или сжатие без изгиба, скорость распространения фронта волны определяется выражением

$$
c^{*}=c\left(1+l_{1} a / 2\right)^{-1},
$$

и амплитуда волны не изменяется. Акустоупругий коэффициент $\beta_{\sigma}\left[{ }^{5}\right]$, связывающий изменение скорости фронта волны с начальным напряжением, определяется из зависимости

$$
\pm\left(c-c^{*}\right) c^{-1}=\beta_{\sigma} \sigma_{22}^{0}
$$

и равняется

$$
\beta_{\sigma}=l_{1} / 2 .
$$


Во втором предельном случае, когда

$$
a=-b X_{1}^{+} / 2 \text {, }
$$

т. е. когда срела испытывает чистый изгиб (напряжения $\sigma_{22}$ в точках $X_{1}=0$ и $X_{1}=X_{1}+$ равны по модулю, но противоположны по знаку) скорость волны в точке наблюдения с координатой $X_{1}{ }^{+}$равна, согласно уравнению (3.8),

$$
c^{*}=c \text {. }
$$

В этом случае единственный искомый параметр $b$ начальной деформашии среды определяется по амплитуде волны формулой

$$
b=4 \Phi_{1}\left[X_{1}^{+}\left(l_{1}+2 l_{2}\right)\right]^{-1} .
$$

\section{Л И Т Е Р А Т У Р А}

1. Гузь А. Н., Махорт Ф.Г., Гуша О. И., Введение в акустоупругость, Киев. «Науковя думка», 1977

2. Г уз ь А. Н., Прикладная механика, 14. № 4. 3-32 (1978).

3. Rera. R. K.. Anl. Mat.. 18. № 1 9-17 (1973).

4. nev. S.. Addv. S. K.. Int. J. Nonlinear Mech., 12, № 3, 371-381 (1977).

5. Бобренко В. М. В ангели М. С., Куценко А. Н., Акустические метолы контроля наппяженного состояния материала деталей машин, Кишинев, «Штиинша». 1981.

6. Нигул У. К., Энгельбрехт Ю. К., В кн.: Эхо-сигналы от упругих объектов. т. 2, Таллин, «Валгус», 1974, с. $233-253$.

Ннститут кибернетики.

Академии наук Эстонской ССР
Поступила в редакцию $19 /$ I 1982

\section{A. RAVASOO}

\section{LAINETE LEVIMINE MITTEHOMOGEENSE STAATILISE ALGDEFORMATSIOONIGA KESKKONNAS}

Artiklis on käsitletud lainete levimist tahkes keskkonnas. mis on algselt tasaninnalise mittehomogeense deformatsiooni olukorras, ia vaadeldud algdeformatsionni määramise võimalust lainete levimist iseloomustavate parameetrite iärgi. On leitud konvolutsiooniintegraali sisaldav lahend. mis võimaldab kirjeldada suvalise kujuga ääretingimuse tekitatud vikilaine kuju evolutsiooni. Pōhialikumalt on uuritud siinusekuiulise ääretingimuse iuhtu. mille puhul konvolutsiooniintegraal avaldub ridadena. On lahendatud akıstodiagnostika ülesanne. määrates siinuslaine abil keskkonna eeldeformatsiooniolukorra, mis lineaarses lähenduses vastab keskkonna puhaspaindele koos tõmbe või survega.

\section{A. RAVASOO}

\section{PROPAGATION OF WAVES IN A MEDIUM WITH AN INHOMOGENEOUS STATICAL INITIAL DEFORMATION}

Propagation of deformation waves in a solid medium with an inhomogeneous statical plane initial deformation is investigated. The main aim of this investigation is to determine the state of the initial deformation using the acoustical methods. The solution of the equation of motion is found in the form of a convolution integral that describes the distortion of the one-dimensional transient longitudinal wave profile. The boundary condition is supposed to be arbitrarv under certain smoothness conditions. The convolution integral is presented in the form of an asvmptotic expansion for the case of sinusoidal boundary condition. This analytical result is used for the determining of the initially deformed state of the medium on the basis of the exnerimental data i. e. an inverse problem of acousto-diagnostics is solved. The case of a plane initial deformation that corresponds to the pure bending with an axial compression or tension is analysed in detail. 